\title{
Unitary braid representations with finite image
}

\author{
MichaEL LARSEN \\ ERIC ROWELL
}

\begin{abstract}
We characterize unitary representations of braid groups $B_{n}$ of degree linear in $n$ and finite images of such representations of degree exponential in $n$.
\end{abstract}

20F36; 20C15

\section{Introduction}

In this paper, we prove two loosely connected results about unitary representations of the braid group $\phi: B_{n} \rightarrow U(d)$, when $n$ is sufficiently large and the degree $d$ is not too large compared to $n$. The original motivation goes back to the work of Jones on images of Braid groups in Hecke algebra representations $H(q, n)$. Jones showed [10] that when $q=i$, the image of $B_{n}$ in every irreducible factor of the Hecke algebra is finite; more explicitly, each such image is an extension of a symmetric group by a 2-group. This is in sharp contrast to the usual behavior of irreducible factors of Hecke algebra representations, in which the closure of the image of $B_{n}$ contains all unimodular unitary matrices (see Freedman, Larsen and Wang [8]). Birman and Wajnryb showed [2] that when $q=e^{2 \pi i / 6}$, certain factors of $H(q, n)$ give rise to representations whose images are extensions of symplectic groups $\operatorname{Sp}\left(2 r, \mathbb{F}_{3}\right)$ by 3 -groups, where $n \approx 2 r$ (see also Goldschmidt and Jones [9]). It seems to be known by some experts, though so far as we know it has not appeared in print, that some other factors of $H\left(e^{2 \pi i / 6}, n\right)$ give rise to image groups which are extensions of $\mathrm{SU}\left(r+1, \mathbb{F}_{2}\right)$ by 2 -groups. Other (extensions of) symplectic groups appear as quotients of the braid group; Wajnryb [19] has found explicit relations exhibiting $\operatorname{Sp}\left(2 r, \mathbb{F}_{p}\right)$ as a quotient of $B_{2 r+1}$ for all $p$. We would like to explain in some sense or at least characterize the possibilities for finite images in such representations. Such a characterization is given in Theorem 4.5.

It appears to be typically the case that a finite image of $B_{n}$ in $U(d)$ can be regarded as a linear group, whose rank is comparable to $n$, over a finite field. We would therefore like to systematically study all representations of $B_{n}$ of dimension $O(n)$ over all fields. Such a study has been initiated for complex representations of degree $\leq n$ by Formanek and his coworkers in $[6 ; 7 ; 17]$. In Theorem 3.3 , we extend these results to 
higher multiples of $n$, but only for unitary representations. For general representations, we have only the very soft result Theorem 2.10, which is used to relate $n$ and $r$ in Theorem 4.5.

Acknowledgements We would like to thank the referee for suggesting several substantive improvements to the paper. Michael Larsen was partially supported by NSF grant DMS-0354772 and Eric Rowell was partially supported by NSA grant H9823008-1-0020.

\section{Braid groups}

In this section we establish some basic facts concerning the braid groups $B_{n}$ and their representations in the general sense of homomorphisms $\phi: B_{n} \rightarrow G$ where $G$ is any group. Proposition 2.2 and Proposition 2.9 can be found in [6], but we include full proofs for the reader's convenience.

For each braid group $B_{n}$ we fix generators $x_{1}, \ldots, x_{n-1}$ such that

$$
\begin{aligned}
x_{i} x_{j} x_{i}=x_{j} x_{i} x_{j} & \text { if }|i-j|=1, \\
x_{i} x_{j}=x_{j} x_{i} & \text { if }|i-j| \neq 1 .
\end{aligned}
$$

Definition 2.1 We say a homomorphism $\phi: B_{n} \rightarrow G$ is constant if

$$
\phi\left(x_{1}\right)=\phi\left(x_{2}\right)=\cdots=\phi\left(x_{n-1}\right) .
$$

Proposition 2.2 If $\phi: B_{n} \rightarrow G$ is a homomorphism and $\phi\left(x_{i}\right)$ commutes with $\phi\left(x_{i+1}\right)$ for some $i \leq n-2$, then $\phi$ is constant.

Proof Applying (2-1) when $j=i+1$, we get

$$
\phi\left(x_{i}\right)^{2} \phi\left(x_{i+1}\right)=\phi\left(x_{i+1}\right)^{2} \phi\left(x_{i}\right),
$$

which implies $\phi\left(x_{i}\right)=\phi\left(x_{i+1}\right)$. As $x_{i}$ commutes with $x_{i+2}, \phi\left(x_{i+1}\right)=\phi\left(x_{i}\right)$ commutes with $\phi\left(x_{i+2}\right)$. By induction on $i$,

$$
\phi\left(x_{i}\right)=\phi\left(x_{i+1}\right)=\cdots=\phi\left(x_{n-1}\right) .
$$

Likewise, $\phi\left(x_{i-1}\right)$ and $\phi\left(x_{i}\right)=\phi\left(x_{i+1}\right)$ commute, so $\phi\left(x_{i}\right)=\phi\left(x_{i-1}\right)$, and by downward induction,

$$
\phi\left(x_{i}\right)=\phi\left(x_{i-1}\right)=\cdots=\phi\left(x_{1}\right) .
$$

Corollary 2.3 If $\phi\left(x_{i}\right) \in Z(G)$ for some $i$, then $\phi$ is constant. 
Corollary 2.4 If $\phi\left(x_{i}\right)=\phi\left(x_{i+1}\right)$ for some $i$, then $\phi$ is constant.

If $j \geq i$, we use the notation $X_{[i, j]}$ for the product $x_{i} x_{i+1} \cdots x_{j}$; if $j<i$, we define $X_{[i, j]}$ to be the identity.

Lemma 2.5 For $k \geq 3$ and $1 \leq i \leq k-2$, we have

$$
X_{[1, k]} x_{i} X_{[1, k]}^{-1}=x_{i+1} .
$$

Proof The lemma holds by the following computation:

$$
\begin{aligned}
X_{[1, k]} x_{i} X_{[1, k]}^{-1} & =X_{[1, i-1]} x_{i} x_{i+1} X_{[i+2, k]} x_{i} X_{[i+2, k]}^{-1} x_{i+1}^{-1} x_{i}^{-1} X_{[1, i-1]}^{-1} \\
& =X_{[1, i-1]} x_{i} x_{i+1} x_{i} x_{i+1}^{-1} x_{i}^{-1} X_{[1, i-1]}^{-1} \\
& =X_{[1, i-1]} x_{i+1} X_{[1, i-1]}^{-1} \\
& =x_{i+1} .
\end{aligned}
$$

Lemma 2.6 If $1 \leq i, j, k, l \leq n-1,|i-j| \geq 2,|k-l| \geq 2$, then there exists $z=z_{i, j, k, l} \in B_{n}$ such that

$$
z x_{i} z^{-1}=x_{k}, z x_{j} z^{-1}=x_{l} .
$$

Proof First we assume $i<j$ and $k<l$. By Lemma 2.5, without loss of generality we may assume $j=l=n-1$. As $X_{[1, n-3]}$ commutes with $x_{n-1}$, the ordered pair $\left(x_{i}, x_{n-1}\right)$ can be conjugated to $\left(x_{i+1}, x_{n-1}\right)$ as long as $1 \leq i \leq n-4$. By induction on $i$, all the $\left(x_{i}, x_{n-1}\right)$ with $i \leq n-3$ are conjugate.

To treat the case that $i>j$ or $k>l$, it suffices to prove that $\left(x_{1}, x_{3}\right)$ can be conjugated to $\left(x_{3}, x_{1}\right)$. Letting

$$
y=x_{1} x_{2} x_{3} x_{1} x_{2} x_{1}=x_{1} x_{2} x_{1} x_{3} x_{2} x_{1},
$$

we have

$$
\begin{aligned}
& y x_{1}=x_{1} x_{2} x_{3} x_{1} x_{2} x_{1} x_{1}=x_{1} x_{2} x_{3} x_{2} x_{1} x_{2} x_{1}=x_{1} x_{3} x_{2} x_{3} x_{1} x_{2} x_{1}=x_{3} y ; \\
& y x_{3}=x_{1} x_{2} x_{1} x_{3} x_{2} x_{3} x_{1}=x_{1} x_{2} x_{1} x_{2} x_{3} x_{2} x_{1}=x_{1} x_{1} x_{2} x_{1} x_{3} x_{2} x_{1}=x_{1} y .
\end{aligned}
$$

Now let $0 \rightarrow A \rightarrow G \rightarrow H \rightarrow 0$ be a central extension. We write $\left[h_{1}, h_{2}\right]^{\sim}$ for the commutator $g_{1} g_{2} g_{1}^{-1} g_{2}^{-1} \in G$, where $g_{i}$ is any element mapping to $h_{i}$. As the extension is central, this is well-defined.

Lemma 2.7 If $0 \rightarrow A \rightarrow G \stackrel{\pi}{\rightarrow} H \rightarrow 0$ is a central extension and $\phi: B_{n} \rightarrow G$ is a homomorphism such that $\pi \circ \phi$ is constant, then $\phi$ is constant. 
Proof Any two elements of $G$ which map to the same element of $H$ must commute. The lemma therefore follows from Proposition 2.2.

Proposition 2.8 If $0 \rightarrow A \rightarrow G \stackrel{\pi}{\rightarrow} H \rightarrow 0$ is a central extension and $\phi: B_{n} \rightarrow H$ is a homomorphism such that $\left[\phi\left(x_{i}\right), \phi\left(x_{j}\right)\right]^{\sim}=1$ for some $i, j$ with $|i-j| \geq 2$, then $\phi$ lifts to a homomorphism $\widetilde{\phi}: B_{n} \rightarrow G$.

Proof As []$^{\sim}$ respects conjugation, Lemma 2.6 implies

$$
\left[\phi\left(x_{i}\right), \phi\left(x_{j}\right)\right]^{\sim}=1
$$

for all $i, j$ with $|i-j| \geq 2$. Fix an element $\tilde{x}_{1} \in G$ with $\pi\left(\tilde{x}_{1}\right)=\phi\left(x_{1}\right)$ and an element $\tilde{y} \in G$ with $\pi(\tilde{y})=\phi\left(X_{[1, n-1]}\right)$. By Lemma 2.5,

$$
\pi\left(\tilde{y}^{k} \tilde{x}_{1} \tilde{y}^{-k}\right)=\phi\left(x_{k+1}\right), k=0,1, \ldots, n-2 .
$$

Let

$$
g_{i}=\tilde{y}^{i-1} \tilde{x}_{1} \tilde{y}^{1-i}
$$

Thus $g_{i}$ and $g_{j}$ commute when $|i-j| \neq 1$, and the elements

$$
a_{i}:=g_{i} g_{i+1} g_{i} g_{i+1}^{-1} g_{i}^{-1} g_{i+1}^{-1}
$$

are all conjugate in $G$ and lie in $A$. Thus, they all coincide; denoting this common element $a$, and setting $\tilde{x}_{i}=a^{i} g_{i}$, we have $\pi\left(\tilde{x}_{i}\right)=\phi\left(x_{i}\right)$, and the $\tilde{x}_{i}$ satisfy the relations (2-1) and (2-2). Defining a homomorphism $\tilde{\phi}$ by the equations $\tilde{\phi}\left(x_{i}\right)=\tilde{x}_{i}$, we see that $\tilde{\phi}$ is a lift of $\phi$.

Proposition 2.9 If $n \geq 5$, then every homomorphism from $B_{n}$ to a solvable group $G$ is constant.

Proof We use induction on the length of the derived series. The proposition follows immediately from Corollary 2.3 when $G$ is abelian, so without loss of generality we may assume that the last nontrivial term $A$ in the derived series of $G$ is a proper subgroup of $G$. By the induction hypothesis, any homomorphism $B_{n} \rightarrow G / A$ is constant. We therefore choose an element $g \in G$ and a sequence $a_{1}, \ldots, a_{n-1} \in Z$ such that $\phi\left(x_{i}\right)=a_{i} g$ for $i=1, \ldots, n-1$. Writing $a^{g}$ for $g a g^{-1}$, we have

$$
a_{i} a_{j}^{g} g^{2}=\phi\left(x_{i} x_{j}\right)=\phi\left(x_{j} x_{i}\right)=a_{j} a_{i}^{g} g^{2}
$$

and therefore

$$
a_{i}^{-1} a_{i}^{g}=a_{j}^{-1} a_{j}^{g}
$$


whenever $|i-j| \geq 2$. The graph on the vertex set $\{1,2, \ldots, n-1\}$ defined by the relation $|i-j| \geq 2$ is connected for $n \geq 5$. Thus,

$$
a_{1}^{-1} a_{1}^{g}=\cdots=a_{n-1}^{-1} a_{n-1}^{g}=a
$$

for some $a \in A$. The braid relation (2-1) for $j=i+1$ implies

$$
a^{3} a_{i}^{2} a_{i+1}=a_{i} a_{i+1}^{g} a_{i}^{g^{2}}=a_{i+1} a_{i}^{g} a_{i+1}^{g^{2}}=a^{3} a_{i} a_{i+1}^{2},
$$

so $a_{1}=\cdots=a_{n-1}$, and $\phi$ is constant as claimed.

We are indebted to the referee for useful suggestions which simplified the proof and improved the constant in the following theorem:

Theorem 2.10 If $\mathcal{G}$ is a linear algebraic group over a field $K$ with solvable component group, and $n \geq \max (5,2 \sqrt{\operatorname{dim\mathcal {G}}}+4)$, then every homomorphism $B_{n} \rightarrow \mathcal{G}(K)$ is constant.

Proof We assume without loss of generality that $K$ is algebraically closed. We use induction on $\operatorname{dim} \mathcal{G}$, the cases $\operatorname{dim} \mathcal{G} \leq 2$ being immediate from Proposition 2.9. We may therefore assume $n \geq 7$. Proposition 2.9 implies also that the composition of $\phi: B_{n} \rightarrow \mathcal{G}(K)$ with the quotient map $\mathcal{G}(K) \rightarrow \mathcal{G}(K) / \mathcal{G}^{\circ}(K)$ is constant. We may therefore assume that $\mathcal{G} / \mathcal{G}^{\circ}$ is cyclic. Assuming without loss of generality that $B_{n} \rightarrow \mathcal{G} / \mathcal{G}^{\circ}$ is surjective, all generators of $B_{n}$ map into the same generator of this cyclic group. If $\mathcal{U}$ denotes the unipotent radical of $\mathcal{G}^{\circ}$, then $\mathcal{U}$ is a normal algebraic subgroup of $\mathcal{G}$. If the composition homomorphism $B_{n} \rightarrow(\mathcal{G} / \mathcal{U})(K)$ is constant, then $B_{n}$ maps to a solvable subgroup of $\mathcal{G}(K)$, namely, an extension of the (cyclic) image of this homomorphism by $\mathcal{U}(K)$. By Proposition 2.9, this implies that $\phi$ is constant. Without loss of generality, therefore, we may assume that $\mathcal{G}$ is reductive. Likewise, composing $\phi$ with the quotient of $\mathcal{G}$ by the center of $\mathcal{G}^{\circ}$, we may assume without loss of generality that $\mathcal{G}^{\circ}$ is adjoint semisimple.

If there exist positive dimensional normal subgroups $\mathcal{N}_{1}, \ldots, \mathcal{N}_{t}$ of $\mathcal{G}$ such that $\mathcal{N}_{1}(K) \cap \cdots \cap \mathcal{N}_{t}(K)=\{1\}$, then the compositions of $\phi$ with the projections $\mathcal{G}(K) \rightarrow$ $\left(\mathcal{G} / \mathcal{N}_{i}\right)(K)$ are all constant, and therefore $\phi$ is constant. If $\mathcal{G}^{\circ}$ has at least two nonisomorphic simple factors, then the product of all factors of any one type is a proper normal subgroup of $\mathcal{G}$. We may therefore assume that $\mathcal{G}^{\circ} \cong \mathcal{H}^{k}$ for some positive integer $k$ and some (adjoint) simple algebraic group $\mathcal{H}$. Moreover, conjugation by a generator of $\mathcal{G} / \mathcal{H}^{k}$ induces a well-defined outer automorphism of $\mathcal{H}^{k}$ and therefore a permutation $\sigma$ of the factors, which are the minimal nontrivial normal subgroups of $\mathcal{H}^{k}$. Without loss of generality we may assume that this permutation is a $k$-cycle, 
since otherwise, each orbit of $\sigma$ determines a product of factors $\mathcal{H}$ which is a normal subgroup of $\mathcal{G}$.

We assume first that $k=1$, so $\mathcal{G}^{\circ}=\mathcal{H}$ is simple. Let $x=\phi\left(x_{n-1}\right)$, and let $B_{n-2}$ denote the subgroup of $B_{n}$ generated by $x_{1}, \ldots, x_{n-3}$. Thus, $\phi\left(B_{n-3}\right)$ lies in the centralizer of $x$ in $\mathcal{G}(K)$. If $x$ is semisimple, setting $\mathcal{K}:=Z_{\mathcal{G}}(x)$, a well-known theorem of Springer and Steinberg [16, Theorem 9.1] implies that the component group of $\mathcal{G}^{\circ} \cap \mathcal{K}$ is commutative and therefore that the component group of $\mathcal{K}$ itself is solvable. If not, let $x_{u} \neq 1$ denote the unipotent factor in the Jordan decomposition $x=x_{u} x_{s}$. Then $x_{u} \in \mathcal{G}^{\circ}(K)$. By the Borel-Tits theorem [3, Proposition 3.1], there exists a parabolic subgroup $\mathcal{P}$ of $\mathcal{G}^{\circ}$ which contains $Z_{\mathcal{G}^{\circ}}\left(x_{u}\right)$ and which is fixed by every automorphism of $\mathcal{G}^{\circ}$ which fixes $x_{u}$. In particular,

$$
Z_{\mathcal{G}}\left(x_{u}\right) \subset N_{\mathcal{G}}(\mathcal{P}) .
$$

As $\mathcal{P}$ is self-normalizing in $\mathcal{G}$, the group $\mathcal{K}:=N_{\mathcal{G}}(\mathcal{P})$ has a solvable component group. In every case, therefore, $\phi\left(B_{n-3}\right)$ lies in $\mathcal{K}$, where $\mathcal{K} / \mathcal{K}^{\circ}$ solvable and $\mathcal{K}^{\circ} \subsetneq \mathcal{G}^{\circ}$. Replacing $\mathcal{K}$ with its quotient by the radical of $\mathcal{K}^{\circ}$, we may assume that $\mathcal{K}^{\circ}$ is a semisimple subquotient of $\mathcal{G}^{\circ}$ From the classification of maximal subgroups by Seitz $[14 ; 15]$ it follows (with some examination of cases) that

$$
\sqrt{\operatorname{dim} \mathcal{K}} \leq \sqrt{\operatorname{dim} \mathcal{G}}-1
$$

so $n-2 \leq \max (5,2 \sqrt{\operatorname{dim} \mathcal{K}}+4)$, and the theorem follows by induction.

Finally, we consider the case $k \geq 2$. Conjugation by $x$ induces an action on $\mathcal{H}^{k}$ given by

$$
\left(h_{1}, \ldots, h_{k}\right) \mapsto\left(\sigma_{1}\left(h_{2}\right), \sigma_{2}\left(h_{3}\right), \ldots, \sigma_{n}\left(h_{1}\right)\right) .
$$

Therefore, the centralizer of $x$ in $\mathcal{G}$ is contained in

$$
\mathcal{K}:=\left\{x^{i}\left(h, \sigma_{1}^{-1}(h), \ldots, \sigma_{n}^{-1} \cdots \sigma_{1}^{-1}(h)\right) \mid 0 \leq i<k, h \in \mathcal{H}\right\} .
$$

Again $\mathcal{K}$ has solvable component group, and

$$
\sqrt{\operatorname{dim} \mathcal{K}}=\sqrt{\operatorname{dim} \mathcal{H}} \leq \frac{\sqrt{\operatorname{dim} \mathcal{K}}}{\sqrt{2}}<\sqrt{\operatorname{dim} \mathcal{G}}-1 .
$$

Again, the theorem follows by induction.

A variant of this idea which will be useful later is the following: 
Proposition 2.11 Let $G$ and $H$ be finite groups, and $k$ and $n$ positive integers, such that $G$ contains a normal subgroup

$$
N \cong \underbrace{H \times H \times \cdots \times H}_{k} .
$$

Suppose the conjugation action of $G$ on $N$ preserves this factorization, and $G / N$ is solvable. If $n \geq \max \left(5,2 \log _{2}|G|\right)$, then every representation $\phi: B_{n} \rightarrow G$ is constant.

Proof Let $f(k)=-2$ for $k=1$ and $f(k)=0$ for $k \geq 2$. We prove that if

$$
n \geq \max \left(5,2 \log _{2}|G|+f(k)\right)
$$

then every $G$-representation of $B_{n}$ is constant. If $G$ is solvable, the theorem is immediate. Otherwise, $|G| \geq 60$, so we may assume $n \geq 9$. We suppose that the ordered quadruple $(G, H, k, n)$ is given and that the proposition is known for all groups of order less than $|G|$. As in the proof of Theorem 2.10, we may assume that $G / N$ is a cyclic group of order $k$ and that $x:=\phi\left(x_{n-1}\right)$ maps to a generator of this quotient. If $k=1$, then $G=H$, and the centralizer $Z_{x}$ of $x$ in $G$ satisfies $\log _{2}\left|Z_{x}\right| \leq \log _{2}|G|-1$. As $\phi\left(B_{n-2}\right) \subset Z_{x}$, and the proposition is known for the quadruple $\left(Z_{x}, Z_{x}, 1, n-2\right)$, we conclude that $\left.\phi\right|_{B_{n-2}}$ is constant, from which it follows that $\phi$ is constant.

If $k \geq 2$, as conjugation by $x$ preserves the decomposition $N \cong H^{k}$, we can write

$$
x\left(h_{1}, \ldots, h_{k}\right) x^{-1}=\left(\sigma_{1}\left(h_{2}\right), \sigma_{2}\left(h_{3}\right), \ldots, \sigma_{n}\left(h_{1}\right)\right)
$$

for automorphisms $\sigma_{i}$. It follows that the centralizer of $x$ is contained in a group $K$ which is an extension of $\mathbb{Z} / k \mathbb{Z}$ by $H$. Applying the induction hypothesis to the quadruple $(K, K, 1, n-2)$, the proposition holds.

\section{Representations of linearly bounded degree}

In this section, we examine the possible degrees of low-dimensional unitary representations of a braid group $B_{n}$. The complex irreducible representations of degree $\leq n$ of $B_{n}$ have been completely described by Formanek et al [7] and Sysoeva [17]. The constant representations have degree 1 , and the nonconstant representations in this range have degree $n-2, n-1$, or $n$. Sysoeva [17] has announced that there are no irreducible representations of degree $n+1$ for $n$ sufficiently large, and has conjectured that such a statement holds for degree $n+k$ as well.

In this section, we consider the irreducible unitary representations of $B_{n}$ of degree $\leq l n$ where $l$ is a fixed integer and $n$ is sufficiently large in terms of $l$. 
We say that a sequence $d_{0}, d_{1}, d_{2}, \ldots$ is weakly convex if the sequence of differences $d_{1}-d_{2}, d_{2}-d_{3}, \ldots$ is nonincreasing.

Lemma 3.1 If $d_{0}, d_{1}, \ldots$ is a weakly convex sequence and $i<j<k$, then there exists an integer $s$ such that

$$
\frac{d_{j}-d_{i}}{j-i} \leq s \leq \frac{d_{k}-d_{j}}{k-j}
$$

Proof Setting $s=d_{j+1}-d_{j}$, the lemma follows immediately.

Lemma 3.2 Let $V$ be a finite-dimensional vector space, $W \subset V$ a subspace, and $T: V \rightarrow V$ an invertible linear transformation. The sequence $d_{0}, d_{1}, d_{2}, \ldots$ defined by $d_{0}:=\operatorname{dim} V$ and

$$
d_{k}:=\operatorname{dim} W \cap T(W) \cap T^{2}(W) \cap \cdots \cap T^{k-1}(W), k \geq 1
$$

is weakly convex.

Proof Define $W_{0}=V$, and

$$
W_{k}:=W \cap T(W) \cap T^{2}(W) \cap \cdots \cap T^{k-1}(W), k \geq 1 .
$$

Then

$$
d_{k}-d_{k+1}=\operatorname{dim} W_{k}-\operatorname{dim} W_{k+1}=\operatorname{dim} W_{k} / W_{k+1}
$$

As $T^{-1}$ maps to $W_{k+1}$ to $W_{k}$ and $W_{k+2}$ to $W_{k+1}$, it induces a map

$$
W_{k+1} / W_{k+2} \rightarrow W_{k} / W_{k+1} .
$$

As

$$
W_{k+1} \cap T\left(W_{k+1}\right)=W_{k+2},
$$

this linear transformation is injective, so

$$
d_{k}-d_{k+1} \geq d_{k+1}-d_{k+2} .
$$

We apply this lemma in the following way. Let $V$ be a finite-dimensional complex vector space endowed with a Hermitian inner product, and $\phi: B_{n} \rightarrow U(V)$ an irreducible unitary representation. For each $\lambda \in \mathbb{C}$, we define $W=W^{\lambda}$ to be the $\lambda$-eigenspace of $\phi\left(x_{1}\right)$. By Lemma 2.5 there exists $y \in B_{n}$ such that $y x_{i} y^{-1}=x_{i+1}$ for $1 \leq i \leq n-2$. We set $T=\phi(y)$. Now, $w \in W^{\lambda}$, if and only if

$$
\left(\phi\left(x_{1}\right)-\lambda\right)(w)=0 .
$$


For any $k$, this is equivalent to

or to

$$
\left(\phi\left(y^{1-k} x_{k} y^{k-1}\right)-\lambda\right)(w)=0,
$$$$
\left(\phi\left(x_{k}\right)-\lambda\right)\left(\phi\left(y^{k-1}\right)(w)\right)=0 .
$$

Thus, the $\lambda$-eigenspace of $\phi\left(x_{k}\right)$ is $T^{k-1}\left(W^{\lambda}\right)$.

We say that an irreducible representation $\phi: B_{n} \rightarrow U(m)$ is of level $k$ if one of the following is true:

(1) $k=0$ and $m=1$.

(2) $k \geq 1$ and $k n-\left(k^{2}+3 k-2\right) \leq m \leq k n$.

Theorem 3.3 For every integer $l \geq 1$ and every integer $n$ sufficiently large in terms of $l$, every irreducible unitary representation of the braid group $B_{n}$ of degree $\leq \ln$ is of some (unique) level $k \leq l$.

\section{Proof As}

$$
(k-1) n<k n-\left(k^{2}+3 k-2\right)
$$

when $n$ is sufficiently large, uniqueness is clear. For existence, we use induction on $l$, the $l=1$ case being known [7]. For given $l \geq 2$, let $\phi: B_{n} \rightarrow \operatorname{Aut}(V)$ be an irreducible unitary representation of degree $\leq \ln$. We may therefore assume that

$$
(l-1) n+1 \leq \operatorname{dim} V \leq \ln -\left(l^{2}+3 l-1\right) .
$$

We write $B_{n-1}$ and $B_{n-2}$ for the subgroups of $B_{n}$ generated by $x_{i}$ with $1 \leq i \leq n-2$ and $1 \leq i \leq n-3$ respectively.

For each eigenvalue $\mu$ of $\phi\left(x_{n-1}\right)$, let $X^{\mu}$ denote the $\mu$-eigenspace. As $B_{n-2}$ commutes with $x_{n-1}, \phi\left(B_{n-2}\right)$ acts on $X^{\mu}$. We say that $X^{\mu}$ splits if it is a direct sum of constant representations of $B_{n-2}$. A sufficient condition that $X^{\mu}$ splits is

$$
\operatorname{dim} X^{\mu} \leq n-5,
$$

as the minimum degree of a nonconstant representation of $B_{n-2}$ is $n-4$. Let $X$ denote the direct sum of all irreducible 1 -dimension factors of $B_{n-2}$ in $V$, so $X$ contains the sum of all split $X^{\mu}$. Let $\lambda_{1}, \ldots, \lambda_{r}$ be the constants appearing in $X$ regarded as a $B_{n-2}$-representation, and let $W^{\lambda_{i}}$ denote the $\lambda_{i}$-eigenspace of $\phi\left(x_{1}\right)$ on $V$, which of course contains the $\lambda_{i}$-eigenspace of $\phi\left(x_{1}\right)$ on $X$. Thus $W_{j}^{\lambda_{i}}$ is the intersection of the $\lambda_{i}$-eigenspaces of $\phi\left(x_{1}\right), \ldots, \phi\left(x_{j}\right)$. As $W_{n-1}^{\lambda_{i}}=\{0\}$, Lemma 3.2 implies

$$
\operatorname{dim} W_{j}^{\lambda_{i}} \geq \frac{n-1-j}{2} \operatorname{dim} W_{n-3}^{\lambda_{i}},
$$


for $1 \leq j \leq n-3$. If $\operatorname{dim} X \geq 2 l+1$,

$$
l n \geq \sum_{i} \operatorname{dim} W_{1}^{\lambda_{i}} \geq \frac{n-2}{2} \operatorname{dim} X \geq \ln +(n / 2-2 l-1) .
$$

Assuming $n>4 l+2$, we may therefore conclude that $\operatorname{dim} X \leq 2 l$.

We consider first the case that there are at least two different eigenvalues $\mu_{i}$ such that $X^{\mu_{i}}$ does not split. For each $\mu \in\left\{\mu_{1}, \ldots, \mu_{r}\right\}$, let $X_{n s}^{\mu}$ denote the orthogonal complement in $X^{\mu}$ of the direct sum of all constant representations of $B_{n-2}$. Then

$$
\begin{aligned}
\operatorname{dim} V-\operatorname{dim} X_{n s}^{\mu} & \leq l n-\left(l^{2}+3 l-1\right)-(n-4) \\
& =(l-1)(n-2)-\left(l^{2}+l-3\right) \\
& <(l-1)(n-2),
\end{aligned}
$$

so $\bigoplus_{\mu_{i} \neq \mu} X_{n s}^{\mu_{i}}$ satisfies the induction hypothesis for representations of $B_{n-2}$, and the same is true of each irreducible factor of each $X_{n s}^{\mu_{i}}$. Each irreducible factor of $X_{n s}^{\mu_{i}}$ therefore has a level. Letting $k_{1}, k_{2}, \ldots, k_{s} \geq 1$ denote the sequence of levels, we have

$$
\operatorname{dim} V=\operatorname{dim} X+\sum_{i=1}^{s} \operatorname{dim} X_{n s}^{\mu_{i}}
$$

so $\left(k_{1}+\cdots+k_{s}\right)(n-2)-\sum_{i=1}^{s} \quad\left(k_{i}^{2}+3 k_{i}-2\right) \leq \operatorname{dim} V \leq 2 l+\left(k_{1}+\cdots+k_{s}\right)(n-2)$.

For $n$ sufficiently large in terms of $l$, this, together with (3-1) implies $k_{1}+\cdots+k_{s}=l$. As $x^{2}+3 x-2$ is convex, for any fixed values of $s \geq 2$ and $l$, the sum of $k_{i}^{2}+3 k_{i}-2$ is minimized, subject to the constraints $k_{i} \geq 1$ and $k_{1}+\cdots+k_{s}=l$, when all but one value of $k_{i}$ is 1 . As the difference between values of $x^{2}+3 x-2$ for consecutive positive integers exceeds the value at $x=1$, if $s$ is constrained to be greater than 1 but otherwise can be chosen freely, the sum of $k_{i}^{2}+3 k_{i}-2$ is maximized when $s=2$. Thus,

$$
\begin{aligned}
\operatorname{dim} V & \geq\left(k_{1}+\cdots+k_{s}\right)(n-2)-\sum_{i=1}^{s}\left(k_{i}^{2}+3 k_{i}-2\right) \\
& \geq \ln -2 l-(l-1)^{2}-3(l-1)+2-2 \\
& =\ln -\left(l^{2}+3 l-2\right) .
\end{aligned}
$$

This leaves the case that there exists a unique $\mu$ such that $X_{n s}^{\mu}$ is not zero. Let $X_{i}^{\mu}$ denote the intersection of the $\mu$-eigenspaces of $x_{n-1}, x_{n-2}, \ldots, x_{n-i}$. By Lemma 3.2, 
applying Lemma 3.1 for $0<i<j$,

$$
\operatorname{dim} X_{i}^{\mu}-\operatorname{dim} X_{j}^{\mu} \leq(j-i)\left\lfloor\frac{\operatorname{dim} V-\operatorname{dim} X_{i}^{\mu}}{i}\right\rfloor .
$$

If

$$
\operatorname{dim} V-\operatorname{dim} X_{l}^{\mu}<l^{2},
$$

then setting $j=n-1$ and $i=l$, we have

$$
\begin{aligned}
\operatorname{dim} V & \leq l^{2}-1+\operatorname{dim} X_{l}^{\mu} \leq l^{2}-1+\operatorname{dim} X_{l}^{\mu}-\operatorname{dim} X_{n-1}^{\mu} \\
& \leq l^{2}-1+(n-l-1)\left\lfloor\frac{l^{2}-1}{l}\right\rfloor \\
& =(l-1) n,
\end{aligned}
$$

which for $n$ sufficiently large is inconsistent with (3-1). On the other hand,

so

$$
\begin{aligned}
& \operatorname{dim} V-\operatorname{dim} X^{\mu} \leq 2 l, \\
& \operatorname{dim} V-\operatorname{dim} X_{l}^{\mu} \leq 2 l^{2} .
\end{aligned}
$$

Assuming that $2 l^{2} \leq n-l-6$, this implies that the orthogonal complement of $X_{l}^{\mu}$ is a split representation of $B_{n-l-1}$, the subgroup of $B_{n}$ generated by $x_{1}, \ldots, x_{n-l-2}$.

Let $\lambda_{i}$ denote the eigenvalues of this representation. We have

$$
\sum_{i} \operatorname{dim} W_{n-l-2}^{\lambda_{i}} \geq l^{2}
$$

On the other hand, $\operatorname{dim} W_{n-1}^{\lambda_{i}}=0$. By Lemma 3.1 and Lemma 3.2,

$$
\operatorname{dim} W_{1}^{\lambda_{i}}-\operatorname{dim} W_{n-l-2}^{\lambda_{i}} \geq(n-l-3)\left\lceil\frac{\operatorname{dim} W_{n-l-2}^{\lambda_{i}}}{l+1}\right\rceil .
$$

As $\lceil x /(l+1)\rceil$ is superadditive in $x$ and $\left\lceil l^{2} /(l+1)\right\rceil=l$,

$$
\begin{aligned}
\sum_{i} \operatorname{dim} W_{1}^{\lambda_{i}} & \geq \sum_{i} \operatorname{dim} W_{n-l-2}^{\lambda_{i}}+(n-l-3)\left\lceil\frac{\operatorname{dim} W_{n-l-2}^{\lambda_{i}}}{l+1}\right\rceil \\
& \geq l^{2}+(n-l-3) l=n l-3 l,
\end{aligned}
$$

contrary to $(3-1)$.

In particular by the proof of Theorem 3.3 we see that $B_{n}$ has no irreducible $(n+1)-$ dimensional unitary representations for $n \geq 16$. The actual lower bound is at least 8 as 
$B_{7}$ has irreducible 8 -dimensional unitary representations (factoring over the Hecke algebra $H(i, 7)$; see Jones [10]).

Theorem 3.3 can be extended to projective unitary representations. In fact, we have the following proposition:

Proposition 3.4 Every irreducible projective unitary representation of $B_{n}$ of degree $d \leq 2^{n / 6}$ lifts to a linear representation of $B_{n}$.

Proof The proposition is trivial for $n \leq 5$. We may therefore assume $n \geq 6$. Thus there exists a sequence $a_{1}<\cdots<a_{2 m}$ of positive odd integers less than $n$, with $m \geq n / 6$. Let $y_{i}=x_{a_{i}}$. The generators $y_{i}$ commute with one another. The central extension

$$
0 \rightarrow U(1) \rightarrow U(d) \stackrel{\pi}{\rightarrow} \operatorname{PSU}(d) \rightarrow 0
$$

defines a commutator map [ $]^{\sim}$. By Lemma 2.6, $\left[\phi\left(x_{i}\right), \phi\left(x_{j}\right)\right]^{\sim}$ is independent of the pair $(i, j)$ provided $|i-j| \geq 2$. It is therefore symmetric as well as antisymmetric and consequently takes values \pm 1 . If $\left[\phi\left(x_{i}\right), \phi\left(x_{j}\right)\right]^{\sim}=1$ for some (and therefore all) $(i, j)$ with $|i-j| \geq 2$, then by Lemma 2.7, $\phi$ lifts to a homomorphism to $U(m)$.

We therefore assume that $\left[\phi\left(y_{i}\right), \phi\left(y_{j}\right)\right]^{\sim}=-1$ for all $i \neq j$. Let

$$
a_{i}=y_{1} y_{2} \cdots y_{2 i-1}, b_{i}=y_{1} y_{2} \cdots y_{2 i-2} y_{2 i} \text {. }
$$

Then

$$
\left[a_{i}, a_{j}\right]^{\sim}=\left[b_{i}, b_{j}\right]^{\sim}=1,\left[a_{i}, b_{j}\right]^{\sim}=(-1)^{\delta_{i j}} .
$$

Let

$$
G_{i}:=\pi^{-1}\left(\phi\left(\left\langle a_{i}, b_{i}\right\rangle\right)\right) .
$$

Clearly, the restriction of the standard representation of $U(m)$ to $G_{i}$ has no 1-dimensional components. The subgroups $G_{1}, \ldots, G_{m} \subset U(d)$ commute in pairs and give rise to a homomorphism $G_{1} \times \cdots \times G_{m} \rightarrow U(d)$. The restriction of the standard representation of $U(m)$ to this product decomposes as a sum of irreducible representations of $G_{1} \times \cdots \times G_{m}$, each of which is an external tensor product of representations of the $G_{i}$, each of degree $>1$. Therefore, $d \geq 2^{m}$.

\section{Representations of exponentially bounded degree}

In this section we fix a constant $c$ and consider nonconstant unitary representations of $B_{n}, n \geq 5$, of degree $d \leq c^{n}$ with finite image. We are interested in the behavior of $G:=\rho\left(B_{n}\right)$. By Proposition 2.9, $G$ cannot be solvable. 
Definition 4.1 We say a finite group $G$ is almost characteristically simple if there exists a (nonabelian) finite simple group $H$ and a positive integer $k$ such that $H^{k}<$ $G<\operatorname{Aut}\left(H^{k}\right)$. We say $G$ is of permutation type if $H$ is isomorphic to the alternating group $A_{n}$ for some $n \geq 5$.

Proposition 4.2 If $G$ is any finite group which is not solvable and $K$ is maximal among normal subgroups of $G$ such that $G / K$ is not solvable, then $G / K$ is almost characteristically simple.

Proof Replacing $G$ by $G / K$, we may assume that $G$ is not solvable but every nontrivial quotient group of $G$ is. In particular, $G$ has no nontrivial normal abelian subgroup. Every minimal normal subgroup $L$ is characteristically simple, ie, of the form $H^{k}$ where $H$ is simple or cyclic of prime order. However, $L$ cannot be abelian, so $H$ cannot be cyclic. If $M$ is any other minimal normal subgroup, it is also a power of a simple group, and $L \cap M=\{1\}$ since $L$ and $M$ are minimal. This implies that the solvable group $G / M$ contains a simple subgroup isomorphic to $H$, which is impossible. It follows that $L$ is the unique minimal normal subgroup, and therefore the conjugation map $G \rightarrow \operatorname{Aut}(L)$ is injective, which proves that $G$ is almost characteristically simple.

Definition 4.3 If $G$ is a finite group which is not solvable, a minimal quotient is any group of the form $G / K$ where $K$ is maximal among normal subgroups of $G$ such that $G / K$ is not solvable.

Definition 4.4 A finite group is of classical type of rank $r$ if it is a finite simple group of the form $A_{r}(q),{ }^{2} A_{r}(q), B_{r}(q), C_{r}(q), D_{r}(q)$, or ${ }^{2} D_{r}(q)$.

Roughly speaking, a finite simple group is of classical type if it is a linear, unitary, orthogonal, or symplectic group over a finite field.

Theorem 4.5 For every constant $c$ there exist positive constants $A, B, K, N$, and $Q$ such that for all $n>N$ and all $\rho: B_{n} \rightarrow U(d)$ with $d \leq c^{n}$ and finite image $G$, every minimal quotient of $G$ is either of permutation type or of the form $H^{k} \rtimes \mathbb{Z} / m \mathbb{Z}$, where $H$ is a finite simple group of classical type of rank $r$. In the latter case, $1 \leq k \leq K$, $2 \leq q \leq Q$, and $A n \leq r \leq B n$.

Proof A minimal quotient is of the form $H^{k} \rtimes C$, where $C$ is solvable and $H$ is simple. By hypothesis, $H$ is not an alternating group. By Proposition 2.11, if $n$ is sufficiently large, then $|H|$ can be taken to be as large as we wish; in particular, we 
exclude that case that $H$ is sporadic. By Theorem 2.10, if $n$ is sufficiently large and $H$ is of Lie type, the dimension of the underlying simple algebraic group must be $>\epsilon n^{2}$ for some absolute constant $\epsilon>0$, so the rank $r$ of the group must be greater than $A n$ for some absolute constant $A>0$. Thus, we may assume that $H$ is a perfect group whose universal central extension is $\mathcal{H}(\mathbb{F})$, where $\mathcal{H}$ is a simply connected semisimple algebraic group over $\mathbb{F}$ which is absolutely simple modulo its center and of rank $r \geq 9$. Let $G_{0}$ denote the inverse image of $H^{k} \subset H^{k} \rtimes C$ in $G$. We have a short exact sequence

$$
0 \rightarrow J \rightarrow G_{0} \rightarrow H^{k} \rightarrow 0,
$$

which we pull back to a short exact sequence

$$
0 \rightarrow J \rightarrow \widetilde{G}_{0} \rightarrow \mathcal{H}(\mathbb{F})^{k} \rightarrow 0 .
$$

As $\widetilde{G}_{0}$ is a central extension of $G_{0}$, the faithful representation $G_{0} \rightarrow U(d)$ gives rise to an almost faithful $d$-dimensional representation of $\widetilde{G}_{0}$. We claim that this implies that $d$ is greater than or equal to the degree of the minimal nontrivial representation of $\mathcal{H}(\mathbb{F})$. Let $X \subset \operatorname{Hom}\left(Z(J), \mathbb{C}^{\times}\right)$denote the set of characters obtained by restricting $\widetilde{G}_{0} \rightarrow U(d)$ to the abelian group $Z(J)$. Thus $\mathcal{H}(\mathbb{F})^{k}$ acts on $X$. If this action is nontrivial, then the permutation representation of $\mathcal{H}(\mathbb{F})^{k}$ acting on $X$ is nontrivial and therefore contains a nontrivial factor. The minimal degree for a nontrivial representation of $\mathcal{H}(\mathbb{F})^{k}$ is the same as that for $\mathcal{H}(\mathbb{F})$. We may therefore assume that $\mathcal{H}(\mathbb{F})^{k}$ acts trivially on $X$. This implies that the action of $\mathcal{H}(\mathbb{F})^{k}$ on $Z(J)$ preserves both $Z\left(\widetilde{G}_{0}\right) \subset$ $Z(J)$ and $Z\left(\widetilde{G}_{0}\right) / Z(J)$ pointwise. As $\mathcal{H}(\mathbb{F})^{k}$ is perfect, any action of this group on an abelian group which fixes a subgroup and quotient group pointwise is trivial. It follows that $Z(J)$ lies in the center of $\widetilde{G}_{0}$. The nonabelian cohomology class which determines whether (4-1) splits lies in $H^{2}\left(\mathcal{H}(\mathbb{F})^{k}, J\right)$, which is a principal homogeneous space of $H^{2}\left(\mathcal{H}(K)^{k}, Z(J)\right)$. The latter is trivial since $\mathcal{H}(K)^{k}$ is centrally closed. Therefore, $G_{0}$ contains a subgroup isomorphic to $\mathcal{H}(\mathbb{F})^{k}$, and restricting $V$ to this subgroup, we see that our claim holds.

The Seitz-Landazuri bound [12] on the minimal degree projective representations of finite simple groups of Lie types now implies that $q^{k r / n}$ is bounded in terms of $c$. Given that $r / n>A$, this gives upper bounds $Q$ and $K$ for $q$ and $k$, and given that $q \geq 2, k \geq 1$, this gives an upper bound $B$ for $r / n$.

We remark that the theorem can be extended in two ways without essentially modifying the proof. On the one hand, we need not assume that the representation $V$ is unitary. On the other hand, if $V$ is unitary, we need not assume that $\rho\left(B_{n}\right)$ is finite; we can take the closure of the image, obtain a compact Lie group, and characterize the group of components of this Lie group without assuming that the identity component is trivial. 


\section{An application}

We would like to describe a general setting in which one obtains sequences of unitary representations of the braid group of exponentially bounded degree. Let $\mathcal{C}$ be any unitary premodular ( = ribbon fusion) category (see Turaev [18, Chapter II.5]). In particular this means that $\mathcal{C}$ is semisimple with finitely many (isomorphism classes of) simple objects $\left\{X_{0}, \cdots, X_{r}\right\}$ and the morphism spaces are finite dimensional $\mathbb{C}$ vector spaces. Moreover, such a category is equipped with a conjugation and a positive definite Hermitian form with respect to which each $\operatorname{End}\left(X^{\otimes n}\right)$ is a Hilbert space. The braiding isomorphisms $c_{X, Y}: X \otimes Y \cong Y \otimes X$ induce unitary representations $\rho_{n}^{X}: B_{n} \rightarrow U\left(\operatorname{End}\left(X^{\otimes n}\right)\right)$ via:

$$
\rho_{n}^{X}\left(\sigma_{i}\right) f=\operatorname{Id}_{X}^{\otimes i-1} \otimes c_{X, X} \otimes \operatorname{Id}_{X}^{\otimes n-i-1} \circ f
$$

for any object $X$, where the $B_{n}$-invariance of the Hermitian form is included in the axioms. By semisimplicity of $\operatorname{End}\left(X^{\otimes n}\right)$ the spaces $\operatorname{Hom}\left(X_{j}, X^{\otimes n}\right)$ for simple $X_{j}$ are equivalent to (potentially reducible) unitary $B_{n}$ subrepresentations of $\operatorname{End}\left(X^{\otimes n}\right)$.

We will show that $\operatorname{dim} \operatorname{Hom}\left(X_{j}, X^{\otimes n}\right)$ is exponentially bounded. For simplicity of notation we assume that $X=X_{i}$ is a simple object and each object is isomorphic to its dual; the general case is essentially the same. For each simple object $X_{i}$ we define a (symmetric) matrix $N_{i}$ whose $(j, k)$-entry is $\operatorname{dim} \operatorname{Hom}\left(X_{k}, X_{i} \otimes X_{j}\right)$. The matrices $N_{i}, 0 \leq i \leq r$ pairwise commute, and are clearly nonnegative. Let $d_{i}$ be the Perron-Frobenius eigenvalue of $N_{i}$, ie the largest eigenvalue. Setting $D=\max \left\{d_{i}\right\}$ we will show that $\operatorname{dim} \operatorname{Hom}\left(X_{j}, X^{\otimes n}\right) \leq D^{n}$. First observe that $d_{i} \geq 1$, since $|\lambda| \leq d_{i}$ for all other eigenvalues $\lambda$ and clearly $\left(N_{i}\right)^{n} \neq 0$ for all $n$. It follows from the Perron-Frobenius Theorem that the vector $\mathbf{d}=\left(d_{0}, d_{1}, \cdots, d_{r}\right)^{T}$ is a strictly positive eigenvector with eigenvalue $d_{i}$ for each $N_{i}$, uniquely determined up to rescaling (one applies the Perron-Frobenius Theorem to the strictly positive matrix $M:=\sum_{i} N_{i}$; see eg Etingof, Nikshych and Ostrik [4]). Now denoting by $\mathbf{e}_{i}$ the $i$-th standard basis vector for $\mathbb{R}^{r}$, we see that $\operatorname{dim}\left(X_{j}, X_{i}^{\otimes n}\right)$ is the $j$-th entry of $\left(N_{i}\right)^{n-1} \mathbf{e}_{i}$ which is less than or equal to the $j$-th entry of $\left(N_{i}\right)^{n-1} \mathbf{d}=\left(d_{i}\right)^{n-1} \mathbf{d}$ which in turn is bounded by $D^{n}$.

There are two well-known constructions of unitary premodular categories. The first is $\operatorname{Rep}\left(D^{\omega} G\right)$ : the representation category of the twisted quantum double of a finite group $G$. $D^{\omega} G$ is a semisimple $|G|^{2}$-dimensional quasi-triangular quasi-Hopf algebra (see Bakalov and Kirillov [1]), and $\operatorname{Rep}\left(D^{\omega} G\right)$ is a modular category. The braid group representations were studied by Etingof, Rowell and Witherspoon [5] and found to have finite images. In particular the image of $\rho_{n}^{H}$ where $H=D^{\omega} G$ is the left regular representation of $D^{\omega} G$ is found to be a subgroup of the full monomial group $S_{n} \ltimes \mathbb{Z}_{s}^{n}$ 
for some $s$ and hence of permutation type. Since any simple object appears as a subobject of $H$, it follows that all images are of permutation type. The second set of examples come from representations of quantum groups at roots of unity (see eg Rowell [13]) or, equivalently, from fixed level representations of affine Kac-Moody algebras. Quantum groups of type $A_{k}$ at 4-th and 6-th roots of unity yield modular categories supporting braid group representations with finite images. In fact, these representations factor over quotients of Hecke algebras $H(q, n)$ and are precisely those alluded to in the introduction. Quantum groups of type $C_{2}$ at 10 -th roots of unity also yield finite braid group images [11], with images $\operatorname{Sp}\left(n-1, \mathbb{F}_{5}\right)$. Here the object $X$ of interest has $d_{X}=\sqrt{5}$, and for $B_{n}$ with $n$ odd, $\operatorname{dim} \operatorname{End}\left(X^{\otimes n}\right)=(\sqrt{5})^{n-1}$ and is the metaplectic representation of $\operatorname{Sp}\left(n-1, \mathbb{F}_{5}\right)$ with two irreducible subrepresentations of dimension $\left((\sqrt{5})^{n-1} \pm 1\right) / 2$. It appears that this can be generalized: there is evidence that quantum groups of type $B_{k}$ at $(4 k+2)$-th roots of unity and $D_{k}$ at $4 k$-th roots of unity support braid group representations with finite symplectic groups as images.

\section{References}

[1] B Bakalov, A Kirillov, Jr, Lectures on tensor categories and modular functors, University Lecture Series 21, Amer. Math. Soc. (2001) MR1797619

[2] J S Birman, B Wajnryb, Markov classes in certain finite quotients of Artin's braid group, Israel J. Math. 56 (1986) 160-178 MR880289

[3] A Borel, J Tits, Éléments unipotents et sous-groupes paraboliques de groupes réductifs. I, Invent. Math. 12 (1971) 95-104 MR0294349

[4] P Etingof, D Nikshych, V Ostrik, On fusion categories, Ann. of Math. (2) 162 (2005) 581-642 MR2183279

[5] P Etingof, E Rowell, S Witherspoon, Braid group representations from twisted quantum doubles of finite groups, Pacific J. Math. 234 (2008) 33-41 MR2375313

[6] E Formanek, Braid group representations of low degree, Proc. London Math. Soc. (3) 73 (1996) 279-322 MR1397691

[7] E Formanek, W Lee, I Sysoeva, M Vazirani, The irreducible complex representations of the braid group on $n$ strings of degree $\leq n$, J. Algebra Appl. 2 (2003) 317-333 MR1997750

[8] MH Freedman, MJ Larsen, $\mathbf{Z}$ Wang, The two-eigenvalue problem and density of Jones representation of braid groups, Comm. Math. Phys. 228 (2002) 177-199 MR1911253

[9] D M Goldschmidt, V F R Jones, Metaplectic link invariants, Geom. Dedicata 31 (1989) 165-191 MR1012438 
[10] V F R Jones, Braid groups, Hecke algebras and type $\mathrm{II}_{1}$ factors, from: "Geometric methods in operator algebras (Kyoto, 1983)", Pitman Res. Notes Math. Ser. 123, Longman Sci. Tech., Harlow (1986) 242-273 MR866500

[11] V F R Jones, On a certain value of the Kauffman polynomial, Comm. Math. Phys. 125 (1989) 459-467 MR1022523

[12] V Landazuri, G M Seitz, On the minimal degrees of projective representations of the finite Chevalley groups, J. Algebra 32 (1974) 418-443 MR0360852

[13] E C Rowell, From quantum groups to unitary modular tensor categories, from: "Representations of algebraic groups, quantum groups, and Lie algebras", Contemp. Math. 413, Amer. Math. Soc. (2006) 215-230 MR2263097

[14] G M Seitz, The maximal subgroups of classical algebraic groups, Mem. Amer. Math. Soc. 67 (1987) no. 365 MR888704

[15] G M Seitz, Maximal subgroups of exceptional algebraic groups, Mem. Amer. Math. Soc. 90 (1991) no. 441 MR1048074

[16] R Steinberg, Endomorphisms of linear algebraic groups, Mem. Amer. Math. Soc. 80, Amer. Math. Soc. (1968) MR0230728

[17] I Sysoeva, Dimension $n$ representations of the braid group on $n$ strings, J. Algebra 243 (2001) 518-538 MR1850645

[18] V G Turaev, Quantum invariants of knots and 3-manifolds, de Gruyter Studies in Math. 18, Walter de Gruyter \& Co., Berlin (1994) MR1292673

[19] B Wajnryb, A braidlike presentation of $\operatorname{Sp}(n, p)$, Israel J. Math. 76 (1991) 265-288 MR1177345

Department of Mathematics, Indiana University Bloomington, IN 47405, USA

Department of Mathematics, Texas A\&M University

College Station, TX 77843-3368, USA

mjlarsen@indiana.edu, rowell@math.tamu.edu

http://mlarsen.math.indiana.edu/ larsen/, http://www.math.tamu.edu/ rowell/

Received: 31 May 2008 Revised: 25 September 2008 